\title{
Review of the literature on de novo formation of cavernous malformations of the central nervous system after radiation therapy
}

\author{
Shahid M. Nimjee, M.D., Ph.D., Ciaran J. Powers, M.D., Ph.D., \\ and Ketan R. Bulsara, M.D. \\ Division of Neurosurgery and Department of Radiology, Duke University Medical Center, Durham, \\ North Carolina
}

\begin{abstract}
$\checkmark$ In this paper the authors review the literature concerning de novo cavernoma formation after radiation treatment. PubMed and MEDLINE database searches were performed. Data were compiled on all patients in whom de novo cavernomas formed after radiation treatment and whose cases were reported in the literature. The authors found reports in the literature of 76 patients in whom cavernomas formed de novo after radiation treatment. The mean age of the patients was 11.7 years, and the majority of these lesions occurred in males. The patients received a mean radiation dose of $60.45 \mathrm{~Gy}$. The mean latency period before detection of the cavernoma was 8.9 years, and most of these lesions were detected incidentally. In symptomatic patients, the most common presenting symptoms were seizures. Thirty-seven of the patients had evidence of hemorrhage, and $54 \%$ of these required surgical intervention.

De novo formation of cavernomas after radiation treatment is a relatively rare phenomenon. Patients in whom these cavernomas develop need to be followed closely because there is a propensity for the lesions to hemorrhage. Surgical intervention to treat symptomatic lesions has a favorable outcome.
\end{abstract}

\section{KEY WORDS • cavernous malformation • cavernoma • brain • spinal cord • radiation therapy}

$\mathrm{T}$ HE use of radiation treatment, either as a primary treatment modality or as an adjunct therapy, is increasing for patients afflicted with central nervous system lesions. Although patients are fortunate in that an alternative treatment option is present, it is becoming clear that there are some long-term sequelae associated with radiation therapy. In this paper, we review the literature regarding radiation-induced cavernomas of the central nervous system.

\section{Review of Databases}

PubMed and MEDLINE database searches were performed. The literature containing reports of de novo formation of cavernomas after radiation treatment was collected.

Abbreviations used in this paper: ALL = acute lymphoblastic leukemia; $\mathrm{CM}=$ cavernous malformation; $\mathrm{MR}=$ magnetic resonance; $\mathrm{WBRT}=$ whole-brain radiation therapy.

\section{Results}

The literature contains reports of 76 patients with de novo formation of cavernomas following radiation treatment (Table 1). Of these, 28 were female and 42 were male (the sex was not reported in six patients). The mean age of the patients was 11.7 years. The primary lesions were as follows: 29 medulloblastoma; 18 glioma; 12 acute lymphoblastic lymphoma; five ependymoma; three pituitary adenoma; two dysgerminoma; and one each for adenoid cystic carcinoma, cavernoma, nasopharyngeal carcinoma, papilloma, and rhabdomyosarcoma. The primary lesion pathology was not specified in two patients. The patients received a mean radiation dose of $60.45 \mathrm{~Gy}$. The mean latency period until detection of the cavernoma was 8.9 years.

Twenty-seven patients had multiple cavernomas. In the 62 patients in whom the information was available, the presenting symptoms of the cavernomas included seizures in $15(24 \%)$, headaches in six $(10 \%)$, and visual disturbances in one $(2 \%)$. Thirty-six patients $(58 \%)$ presented 
TABLE 1

Literature review of de novo formation of CMs in 76 patients after radiation therapy*

\begin{tabular}{|c|c|c|c|c|c|c|c|c|c|}
\hline $\begin{array}{l}\text { Case } \\
\text { No. }\end{array}$ & Primary Tumor & $\begin{array}{l}\text { Age (yrs), } \\
\text { Sex }\end{array}$ & $\begin{array}{c}\text { Total RT } \\
\text { Dose (Gy) }\end{array}$ & $\begin{array}{c}\text { Latency } \\
\text { Post-RT (yrs) }\end{array}$ & Symptoms & $\begin{array}{c}\text { No. of } \\
\text { Lesions }\end{array}$ & Hem & Tx & $\begin{array}{c}\text { Histo } \\
\text { Finding }\end{array}$ \\
\hline 1 & glioma & $11, \mathrm{~F}$ & 70.0 & 6.0 & none & 1 & yes & obs & NA \\
\hline 2 & glioma & $4, \mathrm{~F}$ & 78.0 & 0.417 & seizure & 12 & yes & obs & NA \\
\hline 3 & medulloblastoma & $5, \mathrm{~F}$ & 78.0 & 1.167 & none & 1 & yes & obs & NA \\
\hline 4 & nasopharyngeal & $40, \mathrm{M}$ & 66.0 & 0.417 & none & 1 & yes & obs & NA \\
\hline 5 & medulloblastoma & 4, M & 86.4 & 3.25 & none & 4 & yes & excision & cav \\
\hline 6 & ependymoma & $5, \mathrm{M}$ & 59.5 & 2.417 & none & 1 & yes & obs & NA \\
\hline 7 & astrocytoma & $3, \mathrm{~F}$ & 61.0 & 5.0 & none & 1 & yes & obs & NA \\
\hline 8 & glioma & $13, \mathrm{~F}$ & 52.5 & 22.0 & seizure & 1 & yes & excision & cav \\
\hline 9 & glioma & $9, \mathrm{M}$ & 50.0 & 24.0 & none & 1 & yes & obs & NA \\
\hline 10 & glioma & $19, \mathrm{M}$ & 55.8 & 9.58 & none & 1 & yes & obs & NA \\
\hline 11 & pituitary adenoma & $27, \mathrm{M}$ & 57.6 & 12.0 & seizure & 1 & yes & excision & $\mathrm{cav}$ \\
\hline 12 & medulloblastoma & 3, M & 35.0 & 17.0 & none & 15 & yes & obs & NA \\
\hline 13 & medulloblastoma & $13, \mathrm{M}$ & 55.0 & 0.583 & none & 7 & yes & obs & NA \\
\hline 14 & glioma & $11, \mathrm{M}$ & 51.0 & 6.5 & seizure & $>50$ & yes & excision & cav \\
\hline 15 & glioma & $12, \mathrm{~F}$ & 55.0 & 7.33 & none & $>50$ & yes & excision & cav \\
\hline 16 & ALL & $5, \mathrm{M}$ & 24.0 & 15.0 & none & 6 & yes & obs & NA \\
\hline 17 & adenoid cystic & $21, \mathrm{M}$ & 73.0 & 7.0 & seizure & 2 & yes & excision & cav \\
\hline 19 & astrocytoma & $36, \mathrm{M}$ & 60.0 & 3.0 & none & 1 & yes & obs & NA \\
\hline 20 & glioma & $33, \mathrm{M}$ & 55.0 & 13.0 & none & $>50$ & yes & obs & NA \\
\hline 21 & astrocytoma & $10, \mathrm{~F}$ & 30.0 & 6.0 & headache & NA & NA & excision & cav \\
\hline 22 & pituitary adenoma & $43, \mathrm{~F}$ & 51.0 & 9.0 & none & NA & NA & excision & cav \\
\hline 23 & dysgerminoma & $15, \mathrm{~F}$ & 50.0 & 7.0 & headache & NA & NA & excision & cav \\
\hline 24 & cavernoma & $15, \mathrm{~F}$ & 50.0 & 9.0 & seizure & NA & NA & excision & cav \\
\hline 25 & astrocytoma & $12, \mathrm{M}$ & 54.0 & 3.0 & headache & NA & NA & obs & NA \\
\hline 26 & ALL & $16, \mathrm{M}$ & 24.0 & 2.0 & seizure & NA & yes & excision & $\mathrm{cav}$ \\
\hline 27 & ALL & $3, \mathrm{~F}$ & 18.0 & 8.0 & headache & 2 & yes & obs & NA \\
\hline 28 & ALL & $9, \mathrm{M}$ & 18.0 & 11.0 & vision & 1 & yes & excision & $\mathrm{cav}$ \\
\hline 29 & ALL & $2, \mathrm{M}$ & 18.0 & 12.0 & vertigo/dysarthria & 1 & yes & drainage & NA \\
\hline 30 & ALL & $6, \mathrm{M}$ & 18.0 & 7.25 & seizure & NA & yes & excision & cav \\
\hline 31 & ALL & $2, \mathrm{M}$ & 24.0 & 8.25 & seizure & NA & no & obs & NA \\
\hline 32 & ALL & $7, \mathrm{M}$ & 21.0 & 10.0 & seizure & NA & yes & excision & cav \\
\hline 33 & medulloblastoma & $9, \mathrm{~F}$ & 90.0 & 4.0 & none & NA & no & obs & NA \\
\hline 34 & astrocytoma & $7, \mathrm{~F}$ & 54.0 & 4.0 & headache & NA & yes & excision & cav \\
\hline 35 & glioma & $15, \mathrm{M}$ & 54.0 & 4.0 & none & NA & no & obs & NA \\
\hline 36 & medulloblastoma & $14, \mathrm{M}$ & 54.0 & 3.0 & asymp hem & 1 & yes & excision & cav \\
\hline 40 & medulloblastoma & 5, NA & NA & 9.0 & headache & 1 & no & excision & cav \\
\hline 41 & ependymoma & $2, \mathrm{~F}$ & NA & 14.0 & seizure & 2 & yes & excision & cav \\
\hline 42 & astrocytoma & $2, \mathrm{~F}$ & NA & 19.0 & $\mathrm{CN}$ deficit & $>10$ & yes & excision & cav \\
\hline 43 & astrocytoma & $53, \mathrm{M}$ & 60.0 & 26.0 & seizure & 1 & no & excision & cav \\
\hline 44 & astrocytoma & $53, \mathrm{M}$ & 60.0 & 8.0 & dementia & $>1$ & yes & excision & cav \\
\hline 45 & ALL & $5, \mathrm{NA}$ & $5-32$ & NA & NA & NA & NA & NA & NA \\
\hline 46 & ALL & $5, \mathrm{NA}$ & $5-32$ & NA & NA & NA & NA & NA & NA \\
\hline 47 & rhabdomyosarcoma & $5, \mathrm{NA}$ & $5-32$ & NA & NA & NA & NA & NA & NA \\
\hline 48 & NA & $27, \mathrm{NA}$ & $5-32$ & NA & NA & NA & NA & NA & NA \\
\hline 49 & NA & $29, \mathrm{NA}$ & $5-32$ & NA & NA & NA & NA & NA & NA \\
\hline 50 & ALL & $4, \mathrm{M}$ & 25.0 & 18.0 & NA & 1 & no & obs & NA \\
\hline 51 & medulloblastoma & $9, \mathrm{M}$ & 55.0 & 4.0 & NA & $>1$ & yes & excision & cav \\
\hline 52 & dysgerminoma & $10, \mathrm{M}$ & 50.0 & 6.2 & NA & $>1$ & yes & obs & NA \\
\hline 53 & glioma & $1, \mathrm{M}$ & 50.0 & 16.0 & NA & 1 & no & obs & NA \\
\hline 54 & medulloblastoma & $12, \mathrm{M}$ & 50.0 & 16.2 & NA & 1 & yes & obs & NA \\
\hline 55 & ependymoma & $13, \mathrm{M}$ & 55.0 & 7.5 & NA & 1 & no & obs & NA \\
\hline 56 & ALL & $10, \mathrm{~F}$ & 50.0 & 5.0 & NA & $>1$ & yes & excision & $\mathrm{cav}$ \\
\hline 57 & ependymoma & 7, F & 55.0 & 5.2 & NA & $>1$ & yes & excision & cav \\
\hline 58 & medulloblastoma & $6, \mathrm{~F}$ & 25.0 & 22.0 & NA & $>1$ & no & obs & NA \\
\hline 59 & medulloblastoma & $4, \mathrm{M}$ & 90.0 & 13.5 & none & 2 & NA & obs & NA \\
\hline 60 & medulloblastoma & $8, \mathrm{~F}$ & 112.0 & 13.3 & none & 2 & NA & obs & NA \\
\hline 61 & medulloblastoma & $9, \mathrm{M}$ & 112.0 & 8.3 & none & 2 & NA & obs & NA \\
\hline 62 & medulloblastoma & $6, \mathrm{~F}$ & 111.0 & 8.1 & none & 2 & NA & obs & NA \\
\hline 63 & medulloblastoma & $19, \mathrm{M}$ & 78.0 & 16.1 & none & 2 & NA & obs & NA \\
\hline 64 & medulloblastoma & $4, \mathrm{M}$ & 112.0 & 6.2 & none & 2 & NA & obs & NA \\
\hline 65 & medulloblastoma & $1, \mathrm{~F}$ & 90.0 & 9.5 & none & 2 & NA & obs & NA \\
\hline 66 & medulloblastoma & $4, \mathrm{~F}$ & 78.0 & 3.2 & none & 1 & NA & obs & NA \\
\hline 67 & medulloblastoma & $9, \mathrm{~F}$ & 111.0 & 9.5 & none & 1 & NA & obs & NA \\
\hline 68 & medulloblastoma & $11, \mathrm{~F}$ & 78.0 & 5.0 & none & 1 & NA & obs & NA \\
\hline 69 & medulloblastoma & $2, \mathrm{M}$ & 75.0 & 5.5 & none & 1 & NA & obs & NA \\
\hline 70 & medulloblastoma & $9, \mathrm{M}$ & 78.0 & 2.4 & none & 1 & NA & obs & NA \\
\hline 71 & medulloblastoma & $11, \mathrm{~F}$ & 111.0 & 3.6 & none & 1 & NA & obs & NA \\
\hline 72 & medulloblastoma & $6, \mathrm{M}$ & 92.0 & 5.3 & seizure & 2 & yes & excision & $\mathrm{cav}$ \\
\hline 73 & medulloblastoma & $2, \mathrm{M}$ & 78.0 & 7.5 & none & 1 & NA & obs & NA \\
\hline 74 & medulloblastoma & $10, \mathrm{M}$ & 80.0 & 3.2 & none & 1 & NA & obs & NA \\
\hline 75 & medulloblastoma & $7, \mathrm{~F}$ & 79.0 & 1.1 & none & 1 & NA & obs & NA \\
\hline 76 & medulloblastoma & $13, \mathrm{~F}$ & 111.0 & 4.0 & none & 1 & NA & obs & NA \\
\hline
\end{tabular}

* Asymp = asymptomatic; cav = cavernoma; $\mathrm{CN}=$ cranial nerve; Hem = hemorrhage; Histo = histopathological; $\mathrm{NA}=$ information not available; obs = observation; $\mathrm{RT}=$ radiation therapy; $\mathrm{Tx}=$ treatment. 


\section{De novo formation of CMs in the central nervous system postradiation}

with no symptoms, and symptoms were not reported in 14 patients.

The management method was reported in 71 patients. Twenty-seven $(38 \%)$ underwent excision of their lesion, whereas 44 patients $(62 \%)$ were followed conservatively. The treatment modality was not reported in five patients.

Information regarding hemorrhaging associated with the cavernoma was available in 49 of the 76 patients (the hemorrhagic status of the cavernoma in the other $27 \mathrm{pa}-$ tients was not given). Of the 49 patients in whom information was available, hemorrhaging was associated with the cavernoma in $37(76 \%)$, whereas $12(24 \%)$ had none. Of the patients with hemorrhages, $20(54 \%)$ underwent excision of the cavernoma. Four patients with nonhemorrhagic lesions also underwent excision. All the patients who underwent removal of their lesions reported improvement in their symptoms.

\section{Discussion}

De novo formation of cavernomas after radiation treatment, the majority of which occur in the pediatric population, is observed with increasing frequency. There is a propensity for these lesions to occur in males, and they are most frequently encountered in patients who have undergone radiation treatment for medulloblastomas, gliomas, and ALL. Although the incidence is relatively rare, the clinical implications are significant, with patients requiring close follow up and possible surgical intervention.

In 1994, Gaensler, et al., ${ }^{8}$ reported 20 cases of patients in whom cavernomas developed after radiation treatment for medulloblastomas, ependymomas, astrocytomas, and ALL. The radiation dose ranged from 24 to $78 \mathrm{~Gy}$. The period of latency from radiation treatment to diagnosis of cavernoma was between 5 months and 20 years. All 20 patients were diagnosed based on MR imaging criteria. Of those diagnosed, the pathological examination of the lesion was consistent with cavernoma in six patients.

Pozzati, et al., ${ }^{17}$ reported five pediatric patients in whom cavernomas developed after radiation treatment for cerebral astrocytoma, pituitary adenoma, parasellar dysgerminoma, low-grade glioma, and an occult cavernoma. Patients with these lesions presented with headaches, focal deficits, and seizures. The cavernomas were discovered between 3 and 9 years after radiation treatment on followup MR imaging and were followed with serial imaging. The lesions were resected and confirmed by histological analysis. The pathological features of the occult cavernoma and the de novo formation postradiation were compared, and interestingly, the new cavernoma did not display the mature elements of the occult lesion, which included microhemorrhage, hemosiderin-filled macrophages, and calcification.

Acute lymphoblastic leukemia is the third most common primary tumor associated with radiation-induced de novo formation of cavernomas. Humpl, et al., ${ }^{10}$ reported on four children of a series of 120 who had received 18 to 24 Gy of cranial radiation therapy for treatment of ALL and who presented with seizures, ataxia, and hemiparesis. Analysis with MR imaging and computed tomography scanning revealed intracerebral mass lesions consistent with hemorrhage, and subsequent histological analysis of the resected lesions confirmed the suspected diagnosis of a hemorrhagic $\mathrm{CM}$.

Larson, et al., ${ }^{12}$ reported six cases of radiation-induced cavernomas in three patients who had received WBRT for ALL, in two patients who had received radiation therapy for posterior fossa astrocytomas, and in one patient who had undergone WBRT for medulloblastoma. The three patients who underwent treatment for ALL received 18 or 24 Gy of radiation treatment between the ages of 2 and 7 years. All three of these patients presented with seizures between 7 and 10 years after radiation treatment, and MR imaging revealed features consistent with cavernoma formation and subsequent hemorrhage. Two of the lesions were excised and one was followed with serial MR imaging. In the two patients in whom astrocytomas were diagnosed, one was a 7-year-old girl and the other was a 15year-old boy. Both had undergone resection and $54 \mathrm{~Gy}$ of radiation treatment. Four years posttherapy, one patient presented with headache and vomiting, at which time MR imaging revealed a hemorrhagic cavernoma. In contrast, the other patient was asymptomatic and the cavernoma was found on routine imaging. The symptomatic patient underwent surgery, whereas the asymptomatic one was observed. The final patient was a 9-year-old girl with a posterior fossa medulloblastoma, who was treated with 36 Gy of cerebrospinal and 54 Gy of posterior fossa radiation. Four years after radiation treatment, an MR image revealed a cavernoma with no hemorrhage, and thus the lesion was followed without surgical intervention.

Medulloblastoma is the most common primary tumor for which radiation treatment results in subsequent de novo cavernoma formation. Maeder, et al., ${ }^{14}$ reported a single case of de novo cavernoma formation in a 14-yearold boy who underwent surgery, 36 Gy WBRT, and 54 Gy posterior fossa radiation for treatment of a posterior fossa medulloblastoma. The patient remained disease free until 3 years after treatment, when an MR image revealed a lesion consistent with hemorrhage. Follow-up MR imaging over the next 2 years revealed that the hemorrhage was increasing in size. A total resection of the lesion was performed, and histological analysis revealed a CM. The patient recovered without incident.

Chang, et al., ${ }^{5}$ reported multiple parietal cavernomas in a 13-year-old girl who received radiation therapy after subtotal resection of a choroid plexus papilloma. This patient had presented with worsening seizures. Pathological studies confirmed the diagnosis, and the patient underwent resection of the choroid plexus papilloma, with resolution of her symptoms postoperatively.

Radiation treatment for ependymomas has also been associated with de novo cavernoma formation. Amirjamshidi and Abbassioun ${ }^{2}$ reported on a 7-year-old boy who suffered vertigo and dizziness over several weeks. Computed tomography revealed a posterior fossa midline ependymoma that was also obstructing the fourth ventricle. After subtotal resection of the tumor, the patient received $54 \mathrm{~Gy}$ of radiation to the cerebrospinal axis. Nine years after treatment the patient was free of radiographic or symptomatic signs of tumor recurrence, but he presented with seizures and right hemiparesis. Magnetic resonance imaging revealed a lesion that was hypointense on $\mathrm{T}_{1}$ weighted and hyperintense on $\mathrm{T}_{2}$-weighted images, and that contained flow void regions within. Pathological ex- 

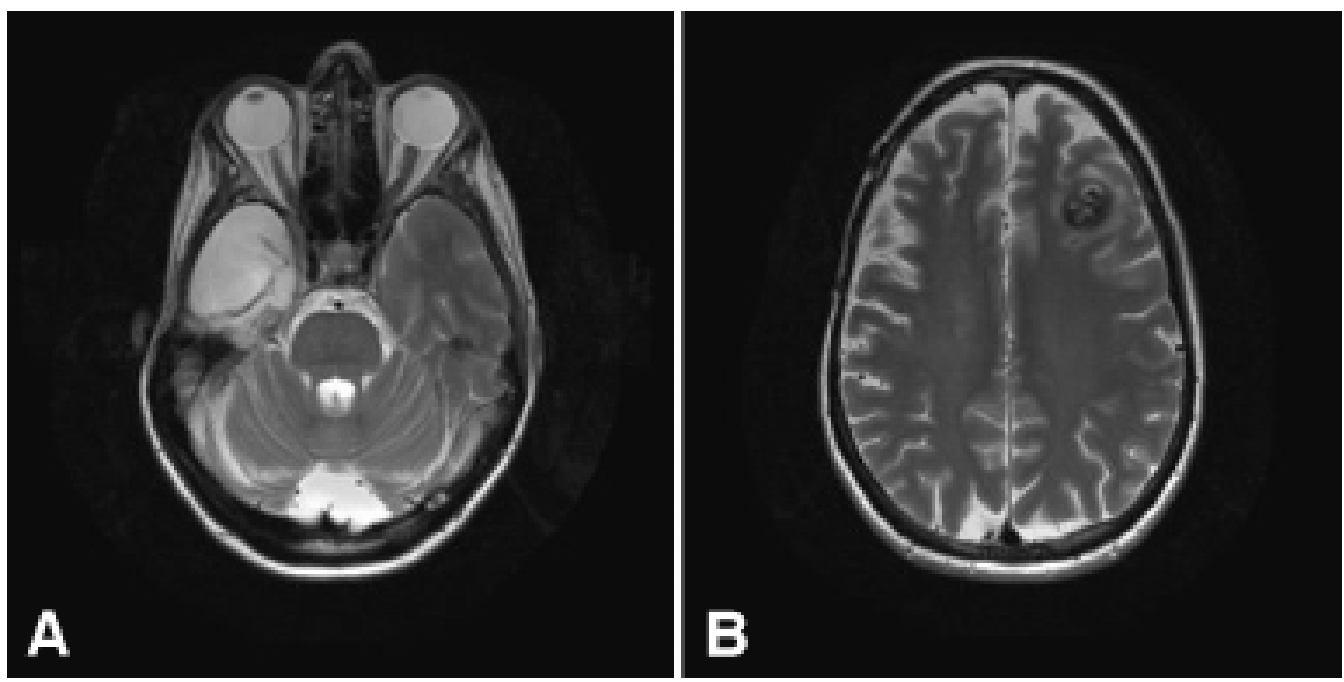

FIG. 1. This 40-year-old woman underwent resection of a right temporal anaplastic astrocytoma in 1997, followed by 46 Gy WBRT. An axial $T_{2}$-weighted MR image obtained in 2006 demonstrates the right temporal resection cavity from the previous surgery and a small cavernoma in the left temporal lobe (left) as well as another cavernoma of the left frontal lobe (right).

amination of the resected lesion revealed a cavernoma. The patient made a remarkable recovery postoperatively.

Baumgartner, et al., ${ }^{3}$ described three cases of radiationinduced cavernomas in which the patients received 45 to 55 Gy of radiation for pediatric brain tumors (medulloblastoma, ependymoma, and midbrain astrocytoma). The lesions appeared 7 to 19 years after radiation treatment and were enlarged on subsequent MR images. Patients presented with seizures, headaches, and focal neurological deficits 1 to 5 years after the lesions were initially noted on MR imaging studies. All three patients underwent microsurgical resection of their lesions between 10 and 21 years after radiation treatment of their primary tumors. Pathological findings in all three lesions were characteristic of cavernomas.

Radiation-induced cavernoma formation usually occurs in children, but it can occur in adults (Fig. 1). In one of the rarer case reports of adult de novo cavernoma formation after radiation exposure, Alexander, et al., ${ }^{1}$ reported a case of a 53-year-old man who presented with syncope 31 years after undergoing a craniotomy and receiving a 31Gy dose of external-beam radiation for a pituitary macroadenoma. At presentation, MR imaging revealed a left frontal cavernoma. The patient was followed and no surgery was performed on the lesion.

The only other report in the literature of radiation-induced cavernoma formation in adults was by Furuse, et al., ${ }^{7}$ who described two 53-year-old men. In the first, a Grade II astrocytoma had been diagnosed, and the patient had received 60 Gy of local brain radiation after subtotal resection of the tumor. Twenty-six years after radiation therapy, he presented with a seizurelike drop attack. An MR image revealed a mass consistent in appearance with a cavernoma, and the lesion was resected. Pathological analysis confirmed the diagnosis. In the second patient, a fibrillary astrocytoma had been diagnosed and was treated with 60 Gy of radiation therapy after subtotal resection of the tumor. Eight years after radiation treatment he began to experience signs of dementia, and MR imaging demonstrated ventricular enlargement. He underwent placement of a ventriculoperitoneal shunt but his symptoms continued to worsen. Three years later, MR imaging revealed an intracerebral hematoma and multiple hyperintense lesions on $\mathrm{T}_{2}$-weighted sequences. The hematoma and lesions were resected, and histopathological analysis confirmed the diagnosis of two cavernomas within the hemorrhagic pocket.

Although most of the literature on radiation-induced de novo formation of cavernomas consists of case reports, Heckl, et al., ${ }^{9}$ presented a case series of 189 cerebral cavernomas diagnosed between 1986 and 2000, five of which had undergone previous radiation. Three of the five patients were 5-year-old children, and the last two were adults who were 27 and 29 years of age at the time of radiation treatment. Two of the children had received radiation for treatment of ALL and the third for treatment of rhabdomyosarcoma of the sphenoid sinus. The two adults had received radiation for unspecified brain tumors. All five patients had received between 5 and 32 Gy of radiation therapy. Heckl, et al., then combined these five cases with 35 others from the literature to investigate the relationship between age and radiation-induced cavernoma formation, and to determine if an association existed between radiation dosage and the latency interval to diagnosis of cavernoma. There was a correlation between radiation-induced cavernomas and children younger than 10 years of age. Cavernoma formation was rare in adults and occurred only in those who had received greater than 30 Gy of radiation.

There are two other case series of cavernoma formation after radiation therapy in childhood. Duhem, et al., ${ }^{6}$ collected nine cases of radiation-induced cavernoma formation. The ratio of boys to girls was $2: 1$. The mean age at diagnosis was 9.8 years. The mean time interval between 


\section{De novo formation of CMs in the central nervous system postradiation}

cranial radiation and diagnosis of cavernoma formation was 11.4 years. Four of the nine patients had a single cavernoma, whereas the other five had between two and eight cavernomas. Hemorrhage was documented in five of the nine cases, whereas only three of the nine patients displayed any symptoms related to the lesion. Three of the patients underwent resection of their malformation. Lew, et al., ${ }^{13}$ focused on radiation-induced cavernoma formation in children treated for medulloblastoma. Fifty-nine patients were followed clinically and radiographically for a mean of 7.2 years from the time of radiation therapy. Their mean age at treatment was 6.6 years and the dose of craniospinal radiation was 24 or $36 \mathrm{~Gy}$, with a posterior fossa radiation boost of 54 or $72 \mathrm{~Gy}$. Of the 59 patients evaluated, cavernomas developed in 18. The mean time from radiation therapy to initial lesion detection on MR imaging was 6.6 years. Twenty of these cases were located in the cerebrum and six were located in the cerebellum. Surprisingly, only one of the 18 patients experienced symptoms related to the lesion's mass effect or hemorrhage, and therefore the lesion was resected in only one patient.

Although the primary focus of our review was intracranial cavernoma formation after radiation treatment, there are case reports of de novo formation of cavernomas after radiation of the spinal cord. Although they appear with equal frequency in cervical and thoracic vertebrae, they are rare in the lumbar region. ${ }^{4}$ The literature has three reports of de novo formation of cavernoma in the spine after radiation treatment. The first case of a spinal cavernoma after spinal axis radiation was presented by Maraire, et al., ${ }^{15}$ who treated a 17 -year-old who originally presented with diabetes insipidus, hypopituitarism, and delayed onset of puberty. The patient's diagnosis was dysgerminoma in the pituitary infundibulum. He underwent resection and received 27 Gy of craniospinal radiation. Five years after therapy, the patient presented with acute onset of thoracic and lumbar back pain, with a band of numbness around his lower abdomen. He also reported bilateral lowerextremity heaviness, constipation, and difficulty urinating. An MR image revealed an intramedullary lesion at T-7 with mild contrast enhancement after addition of gadolinium. After conservative therapy failed, the patient underwent a T-7 hemilaminectomy and costotransversectomy. Pathological analysis of the lesion confirmed the histological features of a cavernoma. Postoperatively, the patient's symptoms resolved, although at the 3-month follow-up evaluation he still required bladder self-catheterization for urinary retention.

Yoshino, et al. ${ }^{18}$ reported a case of a 16-year-old girl who presented with gait abnormality 8 years after receiving spinal radiation for a spinal astrocytoma. An MR image revealed a cavernoma spanning T4-10.

Jabbour, et al., ${ }^{11}$ reported a case of multiple spinal cavernomas in a 33-year-old man who had received radiation therapy as part of his treatment for Wilms tumor 29 years earlier. The cavernoma was discovered incidentally after the patient underwent MR imaging for workup of primary hepatocellular carcinoma. The patient underwent an L1-4 laminectomy and resection of the lesion. Pathological analysis confirmed the diagnosis of cavernoma. The patient recovered uneventfully from surgery.

The pathophysiological mechanisms of radiation-induced cavernoma formation are not clearly understood.
The literature supports the theory that the lesions occur within the radiation field or within the radiation ports. This supports the suggestion that there is no predilection for cavernoma formation in a particular region of the brain. Treatment with WBRT appears to predispose patients to development of cavernoma within the cerebrum rather than in the cerebellum. ${ }^{13}$

There are hypotheses, although they are not well tested, to explain why these lesions occur. Reports have illustrated that the immature brain of the pediatric patient is more sensitive to radiation than the adult brain, and that may account for this disproportionate increase in cavernoma formation. ${ }^{8}$ Radiation therapy can result in vessel wall necrosis and vessel wall changes including cell swelling, dilation of the vessel lumen, hyalinization, fibrosis, and mineralization that predisposes to cavernoma formation. ${ }^{8,10}$ Another group has suggested that the ionizing effect of radiation energy generates vascular and connective tissue changes in the stroma. ${ }^{16}$ Finally, it may be that cavernomas are present before radiation therapy but are radiographically occult at the time of treatment, and it is only with radiation exposure that they can be detected on MR imaging and possibly hemorrhage. ${ }^{12}$

\section{Conclusions}

Insight into understanding the pathophysiological mechanisms of de novo formation of cavernomas may provide valuable information not only for treatment of these lesions once they appear, but also for preventing their manifestation after radiation treatment of brain tumors.

\section{References}

1. Alexander MJ, DeSalles AA, Tomiyasu U: Multiple radiationinduced intracranial lesions after treatment for pituitary adenoma. Case report. J Neurosurg 88:111-115, 1998

2. Amirjamshidi A, Abbassioun K: Radiation-induced tumors of the central nervous system occurring in childhood and adolescence. Four unusual lesions in three patients and a review of the literature. Childs Nerv Syst 16:390-397, 2000

3. Baumgartner JE, Ater JL, Ha CS, Kuttesch JF, Leeds NE, Fuller GN, et al: Pathologically proven cavernous angiomas of the brain following radiation therapy for pediatric brain tumors. Pediatr Neurosurg 39:201-207, 2003

4. Canavero S, Pagni CA, Duca S, Braddack GB: Spinal intramedullary cavernous angiomas: a literature metaanalysis. Surg Neurol 41:381-388, 1994

5. Chang SD, Vanefsky MA, Havton LA, Silverberg GD: Bilateral cavernous malformations resulting from cranial irradiation of a choroid plexus papilloma. Neurol Res 20:529-532, 1998

6. Duhem R, Vinchon M, Leblond P, Soto-Ares G, Dhellemmes P: Cavernous malformations after cerebral irradiation during childhood: report of nine cases. Childs Nerv Syst 21:922-925, 2005

7. Furuse M, Miyatake SI, Kuroiwa T: Cavernous malformation after radiation therapy for astrocytoma in adult patients: report of 2 cases. Acta Neurochir 147:1097-1101, 2005

8. Gaensler EH, Dillon WP, Edwards MS, Larson DA, Rosenau W, Wilson CB: Radiation-induced telangiectasia in the brain simulates cryptic vascular malformations at MR imaging. Radiology 193:629-636, 1994

9. Heckl S, Aschoff A, Kunze S: Radiation-induced cavernous hemangiomas of the brain. A late effect predominantly in children. Cancer 94:3285-3291, 2002 
S. M. Nimjee, C. J. Powers, and K. R. Bulsara

10. Humpl T, Bruhl K, Bohl J, Schwarz M, Stoeter P, Gutjahr P: Cerebral haemorrhage in long-term survivors of childhood acute lymphoblastic leukaemia. Eur J Pediatr 156:367-370, 1997

11. Jabbour P, Gault J, Murk SE, Awad IA: Multiple spinal cavernous malformations with atypical phenotype after prior irradiation: case report. Neurosurgery 55:1431, 2004

12. Larson JJ, Ball WS, Bove KE, Crone KR, Tew JM Jr: Formation of intracerebral cavernous malformations after radiation treatment for central nervous system neoplasia in children. $\mathbf{J}$ Neurosurg 88:51-56, 1998

13. Lew SM, Morgan JN, Psaty E, Lefton DR, Allen JC, Abbott R: Cumulative incidence of radiation-induced cavernomas in longterm survivors of medulloblastoma. J Neurosurg 104 (2 Suppl Pediatrics): 103-107, 2006

14. Maeder P, Gudinchet F, Meuli R, de Tribolet N: Development of a cavernous malformation of the brain. AJNR Am J Neuroradiol 19:1141-1145, 1998

15. Maraire JN, Abdulrauf SI, Berger S, Knisely J, Awad IA: De novo development of a cavernous malformation of the spinal cord following spinal axis radiation. Case report. J Neurosurg 90 (Spine 2):234-238, 1999

16. Nyáry I, Major O, Hanzély Z, Szeifert GT: Histopathological findings in a surgically resected thalamic cavernous hemangioma 1 year after 40-Gy irradiation. J Neurosurg 102 (Suppl):56-58, 2005

17. Pozzati E, Giangaspero F, Marliani F, Acciarri N: Occult cerebrovascular malformations after irradiation. Neurosurgery 39: 677-684, 1996

18. Yoshino M, Morita A, Shibahara J, Kirino T: Radiation-induced spinal cord cavernous malformation. Case report. J Neurosurg 102 (1 Suppl): 101-104, 2005

Manuscript received April 17, 2006.

Accepted in final form May 30, 2006.

Address reprint requests to: Ketan R. Bulsara, M.D., Duke Neurovascular Program, Duke University Medical Center, Box 2601, Durham, North Carolina 27710. email: ketanbulsara@hotmail.com. 